\title{
Timing and Synchronization in Large-Scale Facilities
}

\author{
Franz X. Kärtner ${ }^{(1)}$, Kemal Shafak ${ }^{(2)}$, Ming Xin ${ }^{(3)}$ \\ (1) DESY and Universität Hamburg, 22607Hamburg, Germany, franz.kaertner@desy.de \\ (2) Cycle GmbH, 22607Hamburg, Germany, kemal.shafak@cyclelasers.com \\ ${ }^{(3)}$ DESY and Universität Hamburg, 22607Hamburg, Germany, ming.xin@desy.de
}

\begin{abstract}
We demonstrate sub-femtosecond synchronization of ultrashort pulse lasers and RFsources over $\mathrm{km}$ distances via pulsed optical fiber links. The resulting pulsed fiber network powered by a femtosecond laser with ultralow timing jitter enables clocking of photon-science facilities with attosecond temporal resolution.
\end{abstract}

\section{Introduction}

Large-scale science facilities, such as X-ray free-electron lasers (XFELs) and intense-laser facilities are emerging at a rapid pace around the world. Some of them produce sub-fs X-ray pulses. Therefore, there is a growing need for high-precision timing and synchronization distribution systems, which can synchronize various microwave and optical sources across $\mathrm{km}$-distances with sub-femtosecond precision. Here, we review such a timing distribution and synchronization system utilizing ultralow jitter femtosecond pulse trains from a mode-locked laser transmitted via length stabilized optical fiber links to all positions in a facility that require stable timing. Figure 1 shows the timing

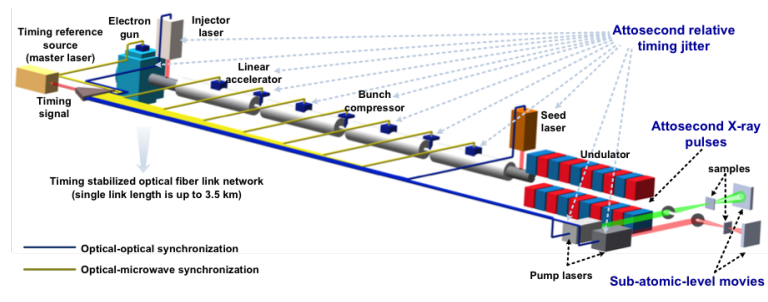

Fig. 1: Timing and synchronization system for an XFEL [1].

distribution system for the case of an XFEL facility. New optical crosscorrelators and balanced optical-microwave phase detectors for improved noise performance have been developed over the last years. Residual secondand third-order dispersion in the fiber links are carefully compensated with additional dispersion-compensating fiber to suppress linkinduced Gordon-Haus jitter and to minimize output pulse duration; the link power is stabilized to minimize the nonlinearity-induced jitter as well as to maximize the signal to noise ratio for locking resulting in attosecond precision across $\mathrm{km}$-scale distances.

Ultralow timing jitter mode-locked lasers

The timing reference source in Fig. 1, also called optical master oscillator (OMO) serves as timing reference for all optical and microwave sources of the network. For sub-femtosecond timing distribution, this laser must exhibit attosecond-level timing jitter in the highfrequency range $(>1 \mathrm{kHz})$, which needs to be confirmed by accurate jitter measurements. We invented the balanced optical cross-correlator method (BOC) [2,3], which is intrinsically immune to AM-PM noise conversion by directly converting the timing difference of two optical pulses into a voltage signal. The BOC characterization has achieved extremely low noise floors down to $10^{-12} \mathrm{fs}^{2} / \mathrm{Hz}$ for offset frequencies up to the Nyquist frequency of mode-locked lasers [4,5].

The OMO jitter characterization setup is shown in Fig. 2. The output of two identical lasers (master and slave, with $216.667 \mathrm{MHz}$ repetition rate, $50 \mathrm{~mW}$ average power, $170 \mathrm{fs}$ pulse width and $1553 \mathrm{~nm}$ center wavelength) have been combined by a polarization beam splitter (PBS) and launched into a BOC, which consists of a single 4-mm-long periodicallypoled KTiOPO4 (PPKTP) crystal operating in a double-pass configuration with appropriate dichroic beam splitter and mirror (DBS, DM) and a low-noise balanced photodetector (BPD). The $B O C$ output was fed back to the piezoelectric transducer (PZT) of the slave laser through a proportional-integral $(\mathrm{PI})$ controller so that the two lasers' repetition rates were locked to each other. Finally, the BOC output was sent to an SSA for jitter characterization.

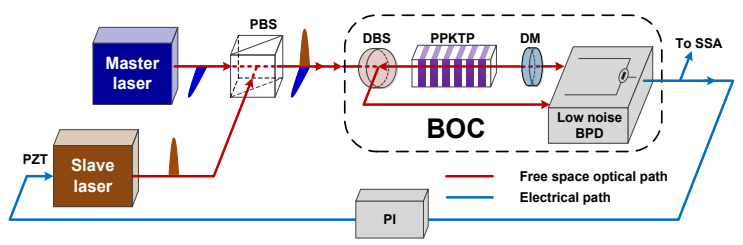

Fig. 2: Master-laser (OMO) jitter characterization setup (PBS, polarization beam splitter; DBS, dichroic beam splitter; DM, dichroic mirror; PPKTP, periodically-poled $\mathrm{KTiOPO}_{4}$; PI, proportional-integral controller; BPD, balanced photodetector; PZT, piezoelectric transducer; SSA, signal source analyzer) [1]. 


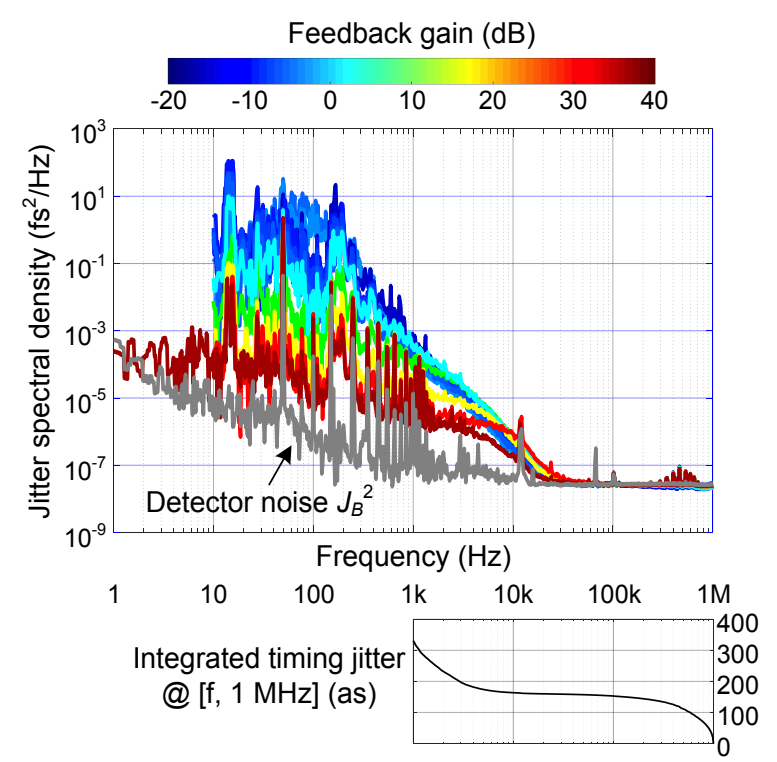

Fig. 3: Measured master-laser jitter spectrum and corresponding integrated timing jitter [6].

The master laser jitter characterization results are displayed in Fig. 3. The top panel shows the jitter spectral density at different feedback gains. It can be seen that with increasing feedback gain, the low frequency timing jitter is suppressed below $50 \mathrm{kHz}$. We can decrease the feedback gain as much as possible (e.g., to -20 $\mathrm{dB}$ ) to obtain an accurate master-laser jitter between $1 \mathrm{kHz}$ and $20 \mathrm{kHz}$ and an upper limit for it above this frequency range.

The jitter spectrum is limited by the detector noise floor (grey curve) above $30 \mathrm{kHz}$. Between $1 \mathrm{kHz}$ and $30 \mathrm{kHz}$, as the gain decreases, the spectrum approaches the real laser jitter. The lowest gain value (about $-15 \mathrm{~dB}$ ) at which the locking is still stable enough to perform a measurement, we obtain 330 as integrated timing jitter from $1 \mathrm{kHz}$ to $1 \mathrm{MHz}$, as shown in the bottom panel of Fig. 3. This value gives a very good estimate for the upper limit of the master laser timing jitter. Thus this laser is definitely capable of providing the reference in an attosecond-precision timing system.

\section{Laser synchronization}

To test the local optical-to-optical synchronization an experimental setup shown in Fig. 4 is constructed. Similar to that in laser characterization, the repetition rates of the slave and master lasers were first locked together with an in-loop BOC, then another out-of-loop BOC was used to evaluate the jitter performance after synchronization. Both BOCs have the same structure as that shown in Fig. 2. In the feedback loop, the output of the in-loop BOC was first filtered by a $\mathrm{PI}$ controller. Then the $\mathrm{PI}$ output was separated into two paths: the first path was directly sent to the slave laser's PZT without amplification to compensate fast jitter above $10 \mathrm{~Hz}$; the second path was sampled by a data acquisition (DAQ) card, analyzed by a Labview program to generate a DC voltage to compensate slow jitter below $10 \mathrm{~Hz}$, and a voltage amplifier was used to extend the compensation range. This feedback design can effectively optimize the locking bandwidth and compensation range simultaneously.

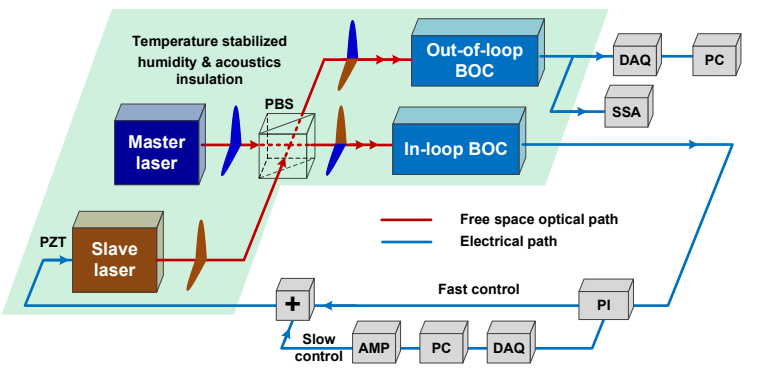

Fig. 4: Local optical-optical synchronization (DAQ, data acquisition card; PC, computer, AMP, voltage amplifier; +, voltage summer) [1].

To minimize the thermally-induced timing fluctuations, the two lasers, two BOCs and other free-space optics were mounted on a temperature-stabilized breadboard with a SuperInvar surface sheet. With temperature fluctuations controlled below $50 \mathrm{mK}$, the effective timing instability of the free-space beam paths due to thermal expansion is only \pm 1 as/cm.

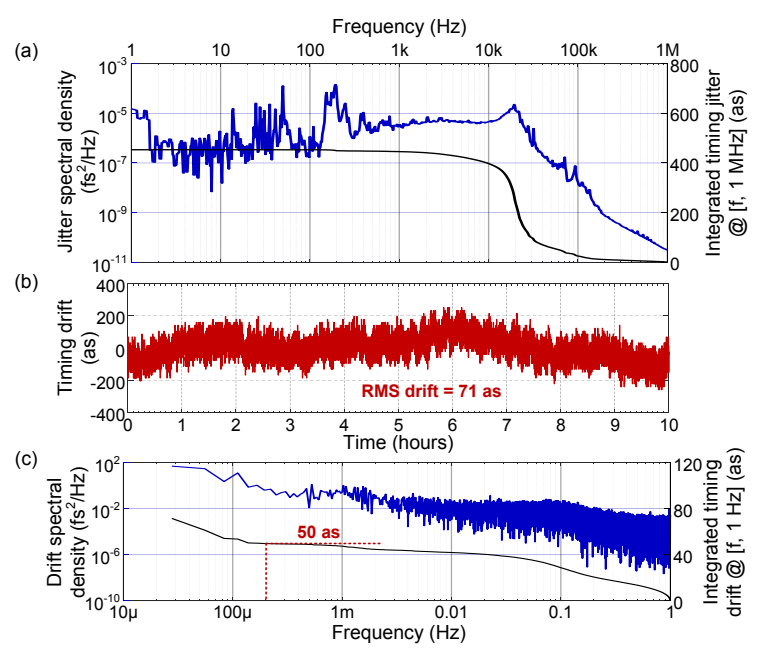

Fig. 5: Local optical-optical synchronization measurement results. (a) Out-of-loop jitter spectrum; (b) long-term timing drift (sampling rate: $2 \mathrm{~Hz}$ ); (c) timing drift spectrum [1].

Fig 5(a) shows the out-of-loop jitter spectrum from $1 \mathrm{~Hz}$ to $1 \mathrm{MHz}$. The total integrated jitter over this frequency range is only 450 as. A longterm drift measurement was taken over 10 hours and the peak-to-peak drift is about 400 as, which gives a root-mean-square (RMS) drift of 71 as (Fig. 5(b)). The Fourier transform of the drift data is also calculated in Fig. 5(c). The integrated drift from $200 \mathrm{mHz}$ to $1 \mathrm{~Hz}$ is only 50 
as. These results indicate that optical synchronization using BOCs can easily achieve attosecond precision.

\section{Microwave Synchronization}

Low noise microwave generation from the output of stabilized fiber links is achieve via Balanced Optical-to-Microwave Phase Detectors (BOMPD) developed in $[7,8]$. The principle of operation of the BOMPD is based on using the pulse train to electro-optically sample the zerocrossings of a microwave signal from a voltage controlled oscillator (VCO). The resulting signal is used to feedback on the VCO frequency thereby disciplining it to a multiple of the $\mathrm{OMO}$ repetition rate.

\section{Sub-femtosecond performance}

Demonstration of sub-femtosecond timing distribution was achieved with the lasermicrowave network shown in Fig. 6a utilizing polarization maintaining and stabilized fiber links [1]. The timing signal from the master laser is distributed through a network that contains two independent fiber links of $1.2-\mathrm{km}$ and $3.5-\mathrm{km}$ length operated in parallel. The link outputs

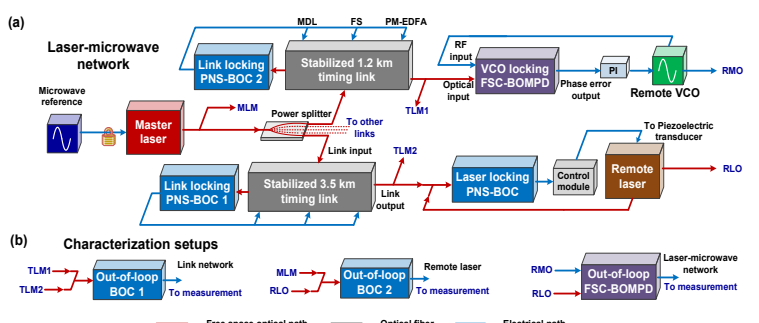

Fig. 6: (a) Laser-microwave network (VCO, voltage-controlled oscillator); (b) Out-of-loop characterization setups [1].

are used to synchronize a remote laser (e.g., serving as a pump-probe laser at the FEL end station) and a voltage-controlled oscillator (VCO) (e.g., serving as a microwave reference of the FEL linear accelerator) simultaneously. New polarization-noise-suppressed BOCs (PNS-BOC) and free-space-coupled balanced optical-microwave phase detectors (FSCBOMPD) for improved noise performance have been implemented. Residual second- and thirdorder dispersion links are carefully compensated with additional dispersion-compensating fiber to suppress link-induced Gordon-Haus jitter and to minimize output pulse duration; the link power is stabilized to minimize the nonlinearity-induced jitter as well as to maximize the SNR for BOC locking. Characterization setups are shown in Fig. $6 \mathrm{~b}$, to evaluate the performance of the link network, as shown in Fig. 7. The residual timing drift between links below $1 \mathrm{~Hz}$ is only 200 as RMS (red), and the total integrated timing jitter from $6 \mathrm{mHz}$ to $1 \mathrm{MHz}$ is 580 as (red). Remote laser synchronization over 44 hours without interruption is within 100 as RMS (blue). Overall, an unprecedented long-term precision of 670 as RMS for the out-of-loop drift over 18 hours (black) is achieved [1].

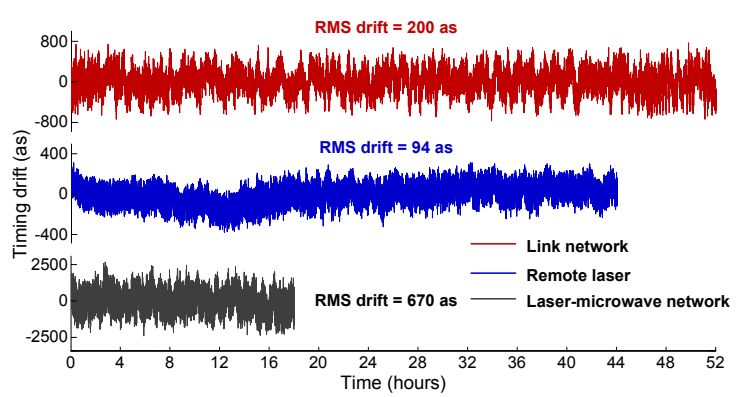

Fig. 7: Measured long-term drift (sampling rate $=2 \mathrm{~Hz}$ ) [1]

\section{Conclusions}

A complete set of ultrafast optical techniques for generating a sub-femtosecond laser-microwave network has been demonstrated using novel timing devices.

\section{Acknowledgement}

This work was supported by the European Research Council under the European Union's Seventh Framework Program (FP/2007-2013) / ERC Grant Agreement No. 609920 and the Cluster of Excellence "The Hamburg Centre for Ultrafast Imaging - Structure, Dynamics and Control of Matter at the Atomic Scale" of the Deutsche Forschungsgemeinschaft.

\section{References}

[1] M. Xin, et al., "Attosecond precision multi-km lasermicrowave network," Light: Science \& Applications, Vol. 6, e16187 (2017).

[2] T. R. Schibli, et al., "Attosecond active synchronization of passively mode-locked lasers by balanced cross correlation," Opt. Lett. Vol. 28, 947 (2003).

[3] J. Kim, et al., "Long-term femtosecond timing link stabilization using a single-crystal balanced cross correlator," Opt. Lett. Vol. 32, 1044 (2007).

[4] J. Kim, et al, "Attosecond-resolution timing jitter characterization of free-running mode-locked lasers," Opt. Lett. Vol. 32, 3519 (2007).

[5] J. Kim, et al., "Drift-free femtosecond timing synchronization of remote optical and microwave sources," Nature Photonics Vol. 2, 733 (2008).

[6] K. Şafak, M. Xin, P. T. Callahan, M. Y. Peng, F. X. Kärtner, All fiber-coupled, long-term stable timing distribution for free electron lasers with few-femtosecond jitter, Structural Dynamics Vol. 2, 041715 (2015).

[7] J. Kim, F. X. Kärtner, F. Ludwig, "Balanced opticalmicrowave phase detectors for optoelectronic phaselocked loops," Opt. Lett. Vol. 31, 3659 (2006).

[8] M. Y. Peng, A. Kalaydzhyan, F. X. Kärtner, Balanced optical-microwave phase detector for sub-femtosecond optical-RF synchronization, Opt. Exp. Vol. 22, 27102 (2014). 